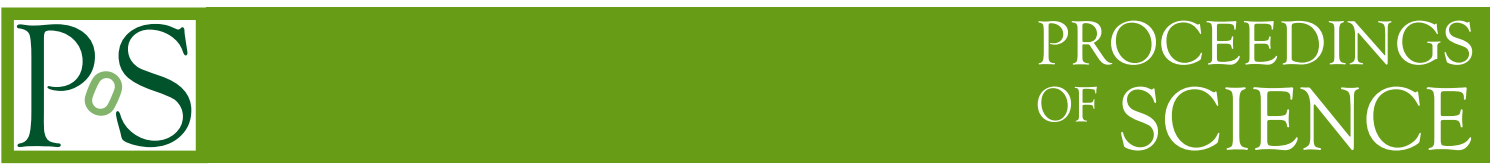

\title{
Status and prospects of the TAx4 experiment
}

\section{Eiji Kido* for the Telescope Array Collaboration ${ }^{\dagger}$}

Institute for Cosmic Ray Research, University of Tokyo, 5-1-5 Kashiwanoha, Chiba 277-8582,

Japan

E-mail: ekidoeicrr.u-tokyo.ac.jp

The TAx 4 experiment is a project to observe highest energy cosmic rays by expanding the detection area of the Telescope Array (TA) experiment with newly constructed surface detectors (SDs) and fluorescence detectors (FDs). The construction of both SDs and FDs is ongoing. New SDs are arranged in a square grid with $2.08 \mathrm{~km}$ spacing at the north east and south east of the TA SD array. Field of view of new FDs overlaps the detection area of new SDs to observe SD FD hybrid events. More than half of the new SDs were deployed on February and March 2019. The first light with new FDs was observed on February 16th, 2018. The performance of the constructed detectors and the prospects of the detector deployment and operation will be shown in this talk as well as proposed physics program. The latter includes high exposure analysis of the hotspot and energy spectrum anisotropy with the extended TA experiment.

36th International Cosmic Ray Conference -ICRC2019-

July 24th - August 1st, 2019

Madison, WI, U.S.A.

\footnotetext{
${ }^{*}$ Speaker.

${ }^{\dagger}$ for collaboration list see PoS(ICRC2019)1177
} 


\section{Introduction}

Telescope Array (TA) is the largest cosmic-ray observatory in the Northern Hemisphere. The TA experiment has 507 surface detectors (SDs) on a square grid with $1.2 \mathrm{~km}$ spacing covering approximately $700 \mathrm{~km}^{2}$. The SDs are surrounded by three fluorescence detector (FD) stations with 12,12 and 14 telescopes. The duty cycle of the SD array is greater than $90 \%$ throughout 10 -year observation period, whereas the FD duty cycle is about $10 \%$ because the data is taken only on moonless clear nights. The locations of the detectors of TA are shown in Fig. 1. The center laser facility (CLF) of TA is located at $39.30^{\circ} \mathrm{N}$ and $112.91^{\circ} \mathrm{W}$ in Utah in the USA.

The hotspot was observed in the arrival directions of 72 cosmic rays with energies above 57 $\mathrm{EeV}$ [2]. The data used in the analysis was obtained by observing with TA SD for 5 years. The chance probability to exceed the obtained maximum significance $(5.1 \sigma)$ in an isotropy sky is estimated to be $3.4 \sigma$ in Ref. [2].

The SD array of the TAx4 experiment was designed to study cosmic rays with the focus on energies above $57 \mathrm{EeV}$. The spacing of TAx4 SD array is sparser than TA SD in favour of larger detection area. The spacing of the array is $2.08 \mathrm{~km}$. Cosmic rays with higher energies make larger number of SDs triggered and the sparse array does not lose the quality of event reconstructions very much at the higher energies. Reconstruction efficiency of the SD array is expected to be more than $95 \%$ above $57 \mathrm{EeV}$. 500 TAx4 SDs cover about 3 times larger area than 507 TA SDs and the combined coverage of TAx4 SDs with TA SDs is about $3000 \mathrm{~km}^{2}$. Fig. 1 shows the planned locations of TAx4 detectors. 2 FD stations of the TAx 4 experiment were also designed. The field of view of FD covers the area of SDs to observe SD FD hybrid events.

Several implications of the energy spectrum anisotropy were recently observed by the TA experiment $[3,4,5]$. The difference between the energy spectrum within 30 degrees from the supergalactic plane and the energy spectrum in the other part of the sky region was evaluated in Ref. [3]. The statistical significance of the difference was estimated to be $3.2 \sigma$. The difference between the energy spectra above and below the declination $\delta=24.8^{\circ}$ was shown in Ref. [4]. The statistical significance of the difference of the break points of the energy spectra was estimated to be $3.5 \sigma$. The energy spectrum anisotropy above $E=10^{19.2} \mathrm{eV}$ was implicated in Ref. [5]. The posttrial significance of the anisotropy was estimated to be $3.7 \sigma$. These implications were obtained from 5-7 year observation of TA SD and are expected to be examined by the TAx4 experiment.

Construction of the TAx4 detectors is now ongoing. The status of the construction of detectors is shown in section 2. Summary and prospects are shown in section 3.

\section{Construction}

The status of the construction of SDs and FDs is shown in section 2.1 and section 2.2, respectively.

\subsection{Construction of SDs}

The detailed design of SDs is shown in Fig. 2 in Ref. [1]. The assembly of scintillator boxes of SDs was started in 2015. The scintillator boxes were assembled in Japan every year and transported to the USA. The assembly in South Korea was also started in 2018. The quality of scintillator boxes 


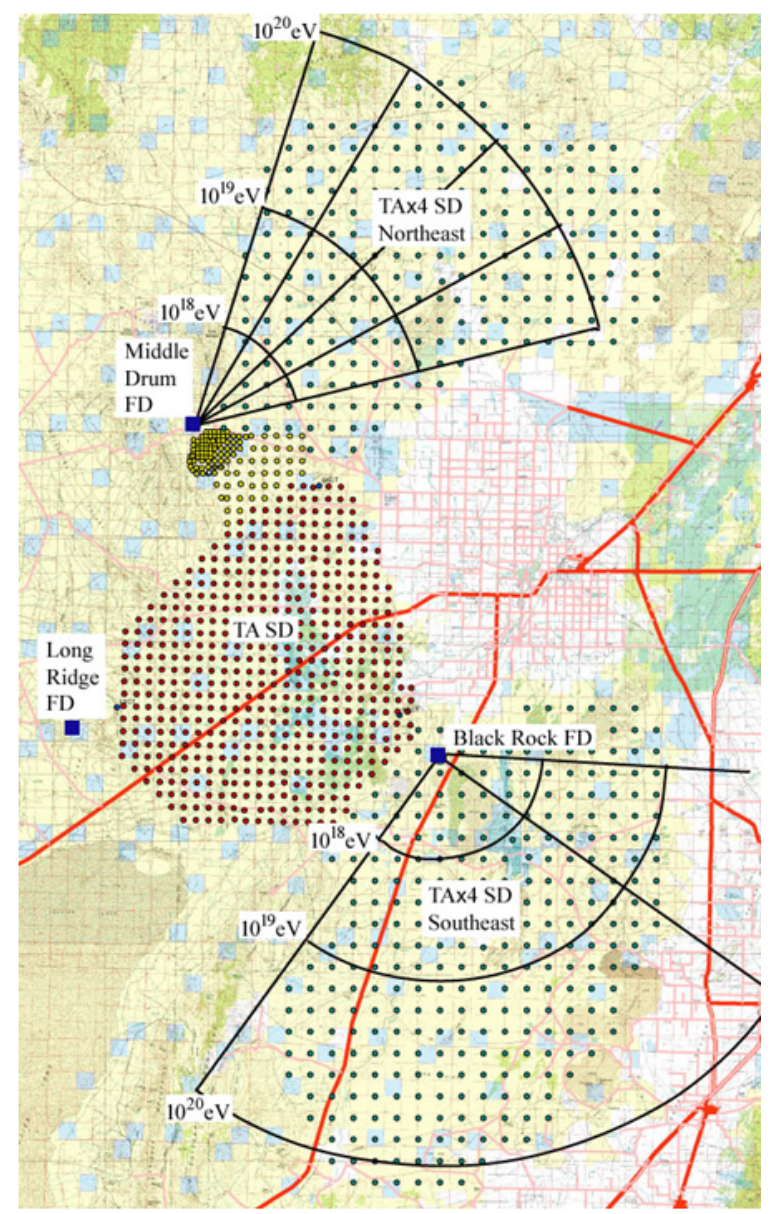

Figure 1: This map shows the overview of the TA site. Each green circle in the northeast and southeast corresponds to the planned location of each TAx4 SD. The spacing of TAx4 SD is $2.08 \mathrm{~km}$. Red circles in the west show the location of TA SD. The spacing of TA SD is $1.2 \mathrm{~km}$. The yellow circle shows the location of TALE SD [6]. The 2 fan shapes drawn with black lines describe the expected field of view of TAx4 FDs. 4 telescopes of FD will be built in the north Middle Drum site and 8 telescopes of FD will be built in the south Black Rock site. The overlap of the locations of SD and the field view of FD enables SD and FD hybrid observation. 


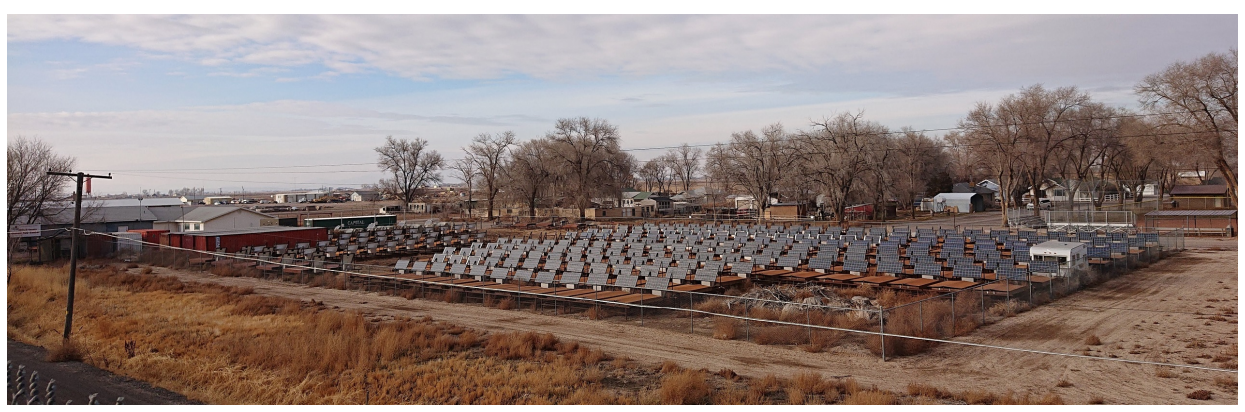

Figure 2: Picture of assembled SDs. These SDs will be deployed in the observation sites.

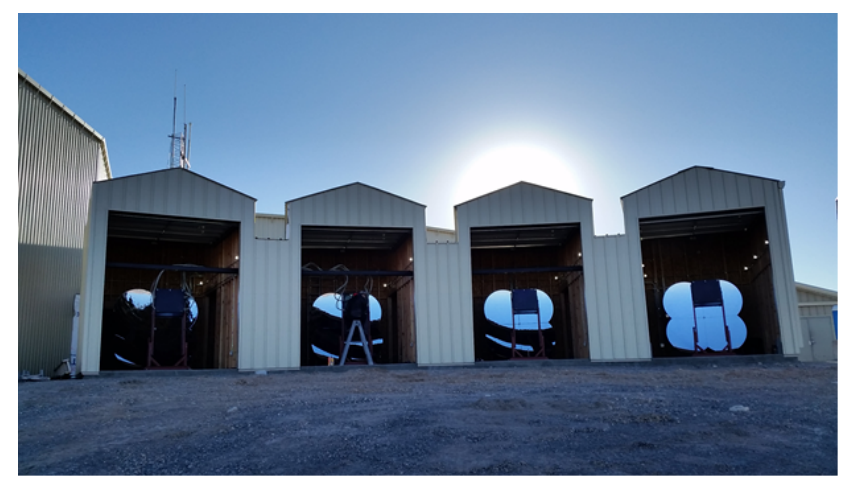

Figure 3: Picture of assembled 4 telescopes in the FD station at the north site of the TAx 4 experiment.

corresponds to the description in section 3 in Ref. [1]. SDs were assembled with the transported scintillator boxes in Delta, Utah. Fig. 2 shows assembled SDs in Delta, Utah. Assembled 257 SDs were deployed on February and March 2019. Fig. 4 shows the locations of deployed SDs. This arrangement of SDs was decided to optimize number of SD FD hybrid events above $10 \mathrm{EeV}$ considering some practical conditions of radio communication between the communication towers and SDs. This SD array is divided into 6 sub-arrays which communicate with different communication towers. Test runs of DAQ at each communication tower were started from the end of April 2019. The same electronics and techniques as the TALE experiment [6] are used for the DAQ. The trigger condition is same as TA SDs [7] except for the gate width. If adjacent 3 SDs with more than 3 MIPs are found within $14 \mu \mathrm{s}$, waveforms with more than $0.3 \mathrm{MIPs}$ from all the SDs in the sub-array are collected within $64 \mu \mathrm{s}$ around the trigger timing. The gate width was changed in order to adjust the larger spacing of the SD array than TA SDs. The DAQ of each sub-array is now being done independently. The boundary trigger between sub-arrays, boundary trigger between TA SD and TAx4 SD and SD FD hybrid trigger will be implemented.

\subsection{Construction of FDs}

North FD station was constructed, and the first light was observed on Feb. 16, 2018. Fig. 3 
shows constructed north FD station. 4 and 8 telescopes are instrumented in the north and south FD station, respectively. Transported telescopes from the HiRes experiment are used for the FD stations. Construction of the south FD station is ongoing.

\section{Summary and Prospects}

More than half of 500 SDs were deployed in the observation sites on February and March 2019. The test run of DAQ of SDs of each sub-array was started from the end of April 2019. The boundary trigger between sub-arrays, boundary trigger between TA SD and TAx4 SD and SD FD hybrid trigger will be implemented. North FD station was constructed, and the south FD station will be constructed soon.

The hotspot and energy spectrum anisotropy will be studied with 4 times larger statistics than TA SDs when the full operation is started. The full operation of the TAx4 experiment will provide about 3 times larger number of SD FD hybrid events than the TA experiment at the highest energies. Mass composition of cosmic rays will be studied in detail with the hybrid events.

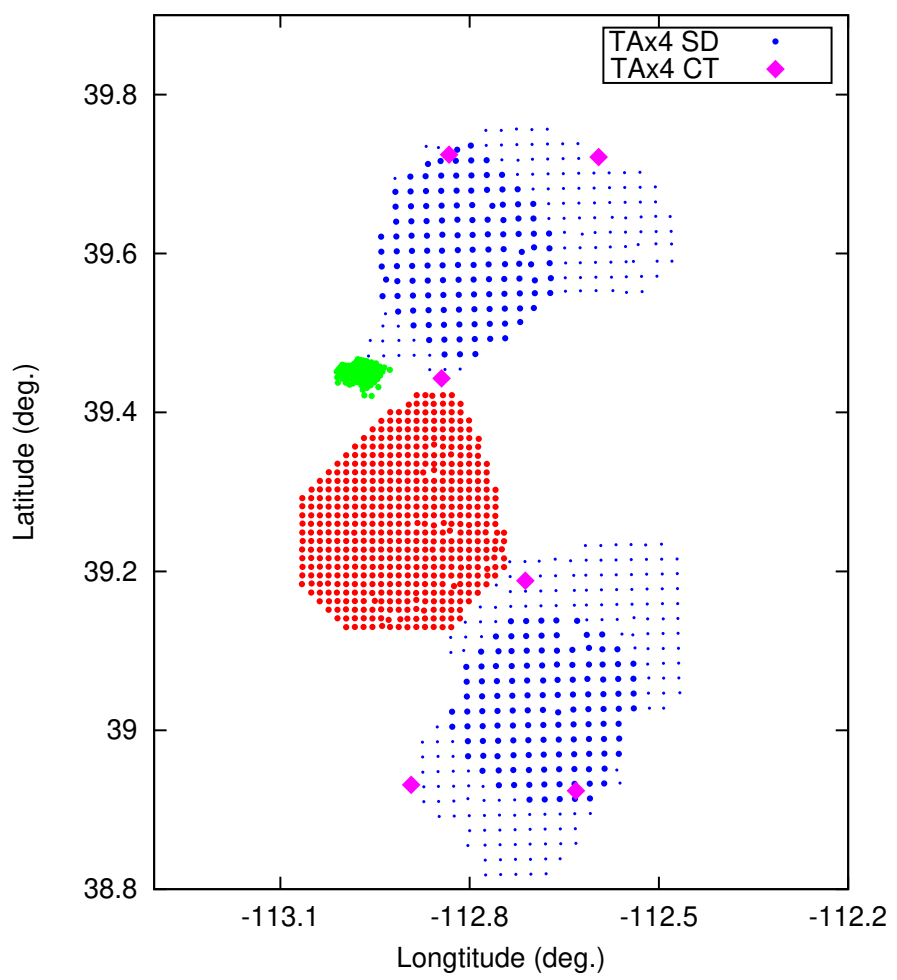

Figure 4: This map shows locations of deployed SDs and constructed communication towers. The thick blue circles are the locations of deployed SDs. The thin blue circles are the locations where SDs were not deployed yet. The pink diamonds are the locations of communication towers. The red circles show the locations of TA SDs. The green circles show the locations of TALE SDs.

\section{References}

[1] E. Kido for the Telescope Array Collaboration, The TAx4 experiment, JPS Conf. Proc. 19, 011025. 
[2] R. Abbasi et al., Indications of Intermediate-scale Anisotropy of Cosmic Rays with Energy Greater Than 57 EeV in the Northern Sky Measured with the Surface Detector of the Telescope Array Experiment, ApJ. 790, L21 (2014).

[3] R. Abbasi et al., Search for Anisotropy in the Ultra High Energy Cosmic Ray Spectrum using the Telescope Array Surface Detector, astro-ph.HE/1707.04967.

[4] R. Abbasi et al., Evidence for Declination Dependence of Ultrahigh Energy Cosmic Ray Spectrum in the Northern Hemisphere, astro-ph.HE/1801.07820.

[5] R. Abbasi et al., Evidence of Intermediate-scale Energy Spectrum Anisotropy of Cosmic Rays E $\geq 10^{19.2} \mathrm{eV}$ with the Telescope Array Surface Detector, ApJ. 862, 91.

[6] S. Udo et al., The Telescope Array Low-energy Extension, in proceedings of International Cosmic Ray Conference, POS ( ICRC2017) 544.

[7] T. Abu-Zayyad et al., The surface detector array of the Telescope Array experiment, NIM-A 689, 87A [astro-ph/1201.4964]. 